\title{
Time evolution of the rule 150 cellular automaton activity from a Fibonacci iteration
}

Jens Christian Claussen

Citation: Journal of Mathematical Physics 49, 062701 (2008); doi: 10.1063/1.2939398

View online: https://doi.org/10.1063/1.2939398

View Table of Contents: http://aip.scitation.org/toc/jmp/49/6

Published by the American Institute of Physics

\section{Articles you may be interested in}

Periodic boundary conditions for QM/MM calculations: Ewald summation for extended Gaussian basis sets The Journal of Chemical Physics 139, 244108 (2013); 10.1063/1.4850655

Arbitrary order El'yashevich-Wilson B tensor formulas for the most frequently used internal coordinates in molecular vibrational analyses

The Journal of Chemical Physics 137, 164103 (2012); 10.1063/1.4759170

The algebraic entropy of classical mechanics

Journal of Mathematical Physics 44, 3071 (2003); 10.1063/1.1576904

Exact Recursion Relation for $2 \times \mathrm{N}$ Arrays of Dumbbells

Journal of Mathematical Physics 11, 3095 (1970); 10.1063/1.1665098

From sequences to polynomials and back, via operator orderings

Journal of Mathematical Physics 54, 123502 (2013); 10.1063/1.4836778

The order of three lowest-energy states of the six-electron harmonium at small force constant

The Journal of Chemical Physics 144, 234304 (2016); 10.1063/1.4953677

\section{Don't let your writing keep you from getting published!}




\title{
Time evolution of the rule 150 cellular automaton activity from a Fibonacci iteration
}

\author{
Jens Christian Claussen ${ }^{a)}$ \\ Institut für Theoretische Physik und Astrophysik, Universität Kiel, Leibnizstraße 15, 24098 \\ Kiel, Germany
}

(Received 5 February 2008; accepted 9 May 2008; published online 27 June 2008)

\begin{abstract}
The rule 150 cellular automaton is a remarkable discrete dynamical system, as it shows $1 / f^{\alpha}$ spectra if started from a single seed [J. Nagler and J. C. Claussen, Phys. Rev. E 71, 067103 (2005)]. Despite its simplicity, a feasible solution for its time behavior is not obvious. Its self-similarity does not follow a one-step iteration like other elementary cellular automata. Here it is shown how its time behavior can be solved as a two-step vectorial, or string, iteration, which can be viewed as a generalization of Fibonacci iteration generating the time series from a sequence of vectors of increasing length. This allows us to compute the total activity time series more efficiently than by simulating the whole spatiotemporal process or even by using the closed expression. The results are further extended to the generalization of rule 150 to the two-dimensional case and to Bethe lattices and the relation to corresponding integer sequences is discussed. () 2008 American Institute of Physics. [DOI: 10.1063/1.2939398]
\end{abstract}

\section{INTRODUCTION}

Since the coining paper of Bak, Tang, and Wiesenfeld (BTW), ${ }^{1}$ there has been considerable interest in the long-time behavior of cellular automata (CA), especially for the occurrence of long range correlations and correspondingly for power spectra exhibiting a power law decay, which have become a paradigm for complex dynamical systems in general. ${ }^{2}$ CAs (Refs. 3 and 4) are a widely studied class of discrete dynamical systems showing emergence of complex spatiotemporal patterns from a simple dynamical rule.

Fibonacci series, defined by $F_{0}=0, F_{1}=1$, and $F_{n}=F_{n-1}+F_{n-2}$, occur in many spatial and temporal patterns from biology to physics, including diffraction, ${ }^{5}$ and mathematical physics gave rise to exotic objects such as Fibonacci anyons. ${ }^{6}$ In this paper another connection is drawn between Fibonacci series and their generalizations and the time series of the total activity of an elementary cellular automaton (ECA).

ECA and sum signals. A cellular automaton consists of an infinite lattice of cells of, e.g., two possible states $(0,1)$, and a local deterministic update rule. At each discrete time step, a cell is updated $x_{n}^{t} \rightarrow x_{n}^{t+1}$ according to the state within a local neighborhood. For Conway's Game of Life ${ }^{7}$ the $3 \times 3$ Moore neighborhood on a two-dimensional (2D) lattice is used. A simpler, yet complex, class is the ECAs, ${ }^{3,4}$ defined on a one-dimensional (1D) lattice, and the update rule depends on the next-neighbor sites and its own state one time step before,

$$
x_{n}^{t+1}=f\left(x_{n+1}^{t}, x_{n}^{t}, x_{n-1}^{t}\right),
$$

where $f$ (the rule) is determined by 8 bits being the output of the possible input bits 000 , $001, \ldots, 111$; this 8 bit number forms the "rule" number which enumerates the 256 possible ECA rules. The power spectra of rule 90 (Ref. 8) and some of the other ECA rules ${ }^{9}$ exhibit a $1 / f^{\alpha}$ decay. Rule 90 and rule 150 can be expressed also as

\footnotetext{
${ }^{\text {a)} E l e c t r o n i c ~ m a i l: ~ c l a u s s e n @ ~ t h e o-p h y s i k . u n i-k i e l . d e . ~}$
} 
TABLE I. Total activity $X\left(t_{1}+t_{2}\right)$ for the first 256 time steps.

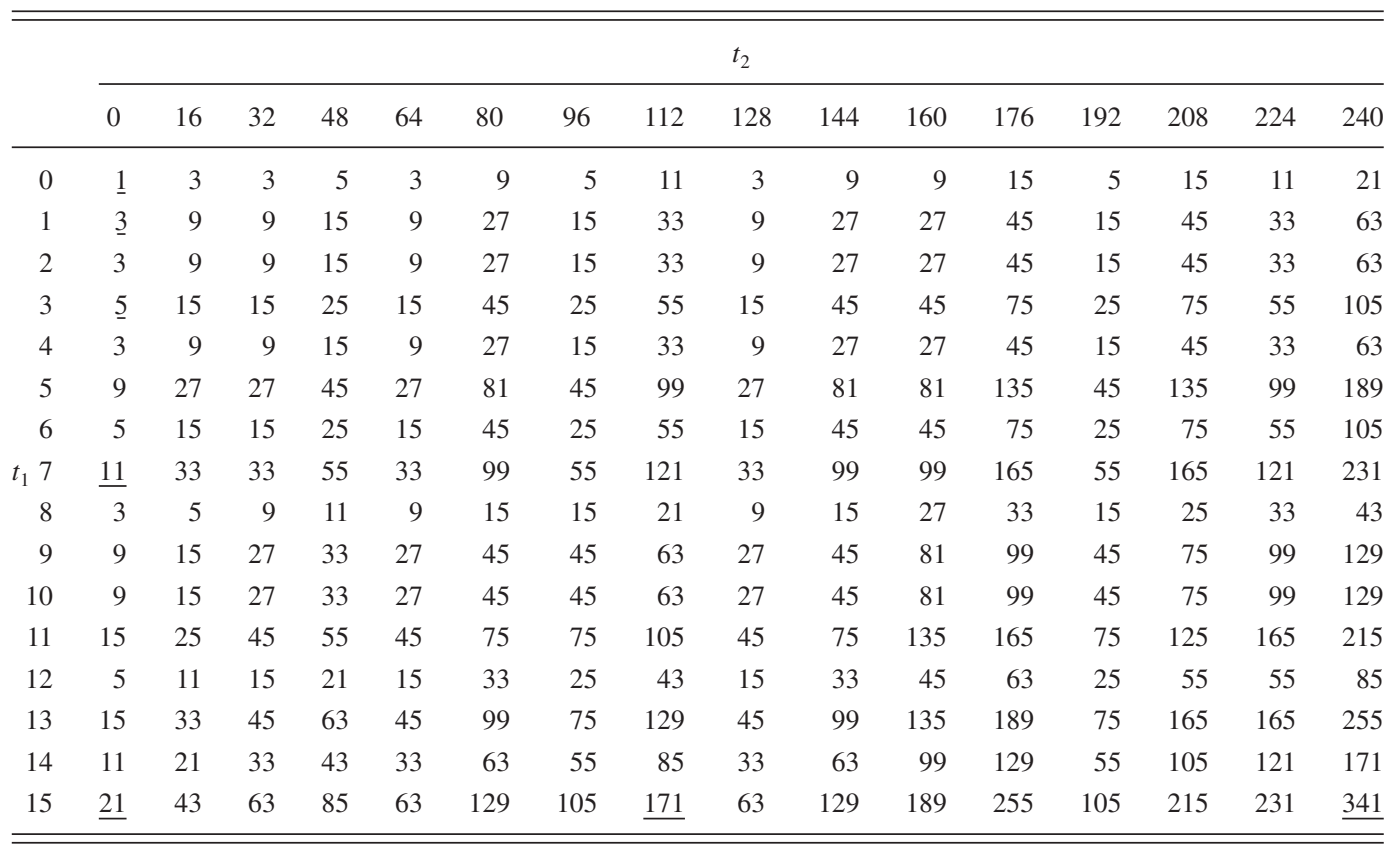

$$
x_{n}^{t+1}=\left[x_{n-1}^{t}+r x_{n}^{t}+x_{n+1}^{t}\right] \bmod 2,
$$

where $r=0$ defines rule 90 , and $r=1$ defines rule 150 , respectively. In the context of catalytic processes, both can be interpreted as local self-limiting reaction processes. ${ }^{8,9}$ As models for chemical turbulence, similar dynamics with a continuous phase variable have been discussed in Refs. 10 and 11, including solitonic behavior, periodic, and turbulent states.

Total activity. In the chemical picture, the total reaction rate at a given time $t$ corresponds to the total number of sites with $x_{m}^{t}=1$, described by the sum signal

$$
X(t)=\sum_{n} x_{n}^{t} .
$$

While for rule 150 there is no convenient solution of $X(t)$ except a formal one, ${ }^{12}$ and a fairly complicated expression (see Sec. II), it is computationally quite costly to perform the full spatiotemporal dynamical simulation, even if one is interested only in the time series. This paper gives an iterative solution of $X(t)$ from a geometrical iteration and investigates the relationship to the Fibonacci iteration. The block sums over $0 \leqslant t \leqslant 2^{N}-1$ can even be expressed directly via Fibonacci numbers. Throughout this paper the pure patterns generated by a single 1 are considered on an infinite lattice.

\section{EXACT SOLUTION}

While in the Sierpinski (rule 90) case $X(t)$ factorizes in a product of $X_{i}\left(\sigma_{i}\right)$ for all "time spins," ${ }^{, 8}$ for rule 150 it does not. However, again, a "spin decomposition" of time $t=\sum_{j=0}^{N-1} \sigma_{j}{ }^{j}$ with $\sigma_{j} \in\{0,1\}$ can be utilized as an efficient coordinate system for the time axis.

Before turning to the geometric iteration, it should be mentioned that a closed expression, in fact, can be written down as follows. As pointed out by Wolfram, ${ }^{4}$ for rule 150 the "correlation" of the time spins comes into play, i.e., $X(t)$ is exactly multiplicative for blocks of spins of value 1 which are separated by one or more zeros. Then $X(t)=\prod_{n=1}^{N} \chi(n)^{c_{n}}$, where $c_{n}$ is the multiplicity of blocks of length $n$. The series $\chi(n)$ (Ref. 13) follows the iteration $\chi(n)=2 \chi(n-1)-(-1)^{n}$ for $n$ $\geqslant 1$ and $\chi(0)=1$. Obviously $\chi(n)=X\left(2^{n}-1\right)$ holds, see underlined entries in Table I. The $\chi(n)$ on 


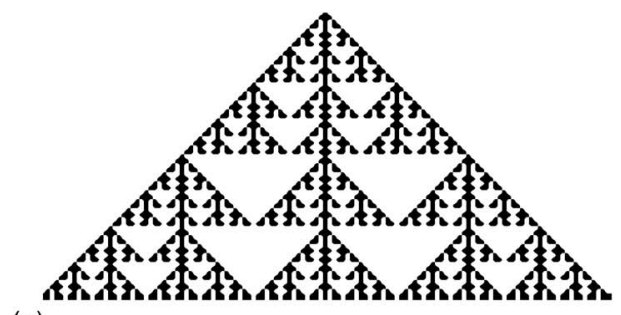

(a)

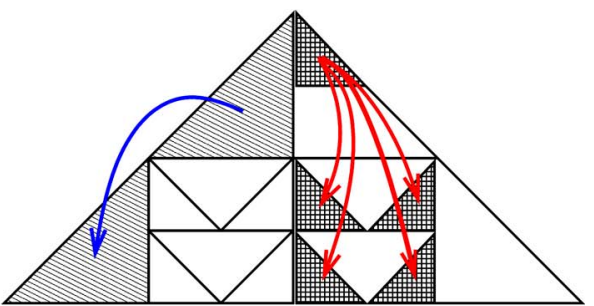

(b)

FIG. 1. (Color online) (a) Time evolution of rule 150 for the first 64 time steps, started with a single seed. (b) Illustration of the replication rule. The whole system is symmetric with respect to the vertical axis. The whole triangle above is replicated once (left part of the triangle). The upper part is reproduced quadruplicate (right part of the triangle).

the other hand turn out as the most decaying frequencies in the spectrum of rule 90 (see Fig. 2 in Ref. 8) and can be expressed in closed form by

$$
\chi(n)=\left\lfloor\frac{2^{n+2}+1}{3}\right\rfloor,
$$

where \lfloor\rfloor is the floor function. The Jacobsthal sequence $\chi(n)$ describes the number of ways to tile a $3 \times(n-1)$ rectangle with $1 \times 1$ and $2 \times 2$ square tiles. ${ }^{14,15}$ Defining $\sigma_{-1}:=0$ and $\sigma_{N}:=0$, one can formalize the spin-block counting as

$$
X(t)=\prod_{n=1}^{N} \chi(n)\left(\Sigma_{i=0}^{N-n}\left(1-\sigma_{i-1}\right)\left(1-\sigma_{i+n}\right) \Pi_{l=0}^{n-1} \sigma_{i+l}\right) .
$$

For example, $t=43$ has the binary representation $\left(\sigma_{5}, \sigma_{4}, \sigma_{4}, \sigma_{2}, \sigma_{1}, \sigma_{0}\right)=(1,0,1,0,1,1)$, thus containing two blocks of length 1 and one block of length 2; hence $X(43)=(\chi(1))^{2} \chi(2)=3^{2} \cdot 5=45$. With our expression (4) and (5) is a closed solution and corresponds to $X_{90}(t)=\Pi_{j=0}^{N-1} 2^{\sigma_{j}}$ in the Sierpinski case. Due to the complicated time spin correlations, it, however, looks quite unwieldy for analytical use and even is numerically unfeasible. ${ }^{16}$

\section{ITERATIVE SOLUTION BY GENERALIZED HYPER-FIBONACCI SERIES}

In contrast to the rule 90 (Sierpinski) case, for rule 150 the time evolution does not follow the same type of initiator-generator mechanism as it is well known for fractal sets. However, it is possible to define a geometric or measure-theoretic ${ }^{17}$ iteration based on the last and the last but one iterate, see Fig. 1. This corresponds to a difference equation with the right hand side depending on the last two time steps and, in fact, for the total activity within $2^{N}$ time steps we will derive a difference equation later.

According to the replication law (see Fig. 1), the time series of the total activity $X(t)$ follows the two-step iteration

$$
\begin{gathered}
X\left(1,0, \sigma_{n-3} \cdots \sigma_{0}\right)=+3 X\left(0,0, \sigma_{n-3} \cdots \sigma_{0}\right), \\
X\left(1,1, \sigma_{n-3} \cdots \sigma_{0}\right)=2 X\left(0,0, \sigma_{n-3} \cdots \sigma_{0}\right)+X\left(0,1, \sigma_{n-3} \cdots \sigma_{0}\right) .
\end{gathered}
$$

At this point a vector notation for the first $2^{n}$ time steps of the time series,

$$
\mathbf{Y}_{n}:=(X(0,0, \ldots, 0,0), X(0,0, \ldots, 0,1), X(0,0, \ldots, 1,0), \ldots X(1,1, \ldots, 1,1))^{T},
$$

i.e., $\left(\mathbf{Y}_{n}\right)_{t}=X\left(\sigma_{n-1}, \sigma_{n-2}, \ldots \sigma_{0}\right)$ with $t=\sum_{j=0}^{N-1} \sigma_{j} 2^{j}$, becomes useful. In this vector notation, (6) reads (Fig. 2) 


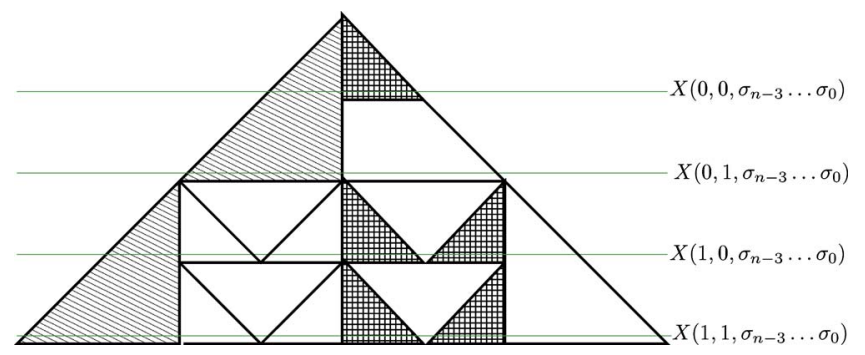

FIG. 2. (Color online) Concept of the "time spin" coordinates. The two leading binary digits of $t$ fix the quarter of the time interval. The remaining digits address the location within each interval.

$$
\begin{aligned}
& \left(\mathbf{Y}_{n}\right)= \\
& \left(\begin{array}{l}
\mathbf{Y}_{n-1} \\
\mathbf{Z}_{n-1}
\end{array}\right), \\
& \left(\mathbf{Z}_{n}\right)=\left(\begin{array}{ll}
3 \cdot \mathbf{1} & \mathbf{0} \\
2 \cdot \mathbf{1} & \mathbf{1}
\end{array}\right)\left(\begin{array}{l}
\mathbf{Y}_{n-1} \\
\mathbf{Z}_{n-1}
\end{array}\right) .
\end{aligned}
$$

The initial vector is given by

$$
\left(\begin{array}{l}
\mathbf{Y}_{0} \\
\mathbf{Z}_{0}
\end{array}\right)=\left(\begin{array}{l}
1 \\
3
\end{array}\right)
$$

Equations (8) and (9) can be collected together to the iteration

$$
\left(\begin{array}{c}
\mathbf{Y}_{n} \\
\mathbf{Z}_{n}
\end{array}\right)=\left(\begin{array}{cc}
\mathbf{1} & \mathbf{0} \\
\mathbf{0} & \mathbf{1} \\
3 \cdot \mathbf{1} & \mathbf{0} \\
2 \cdot \mathbf{1} & \mathbf{1}
\end{array}\right)\left(\begin{array}{l}
\mathbf{Y}_{n-1} \\
\mathbf{Z}_{n-1}
\end{array}\right)
$$

where the dimension of the vectors $\mathbf{Y}_{n}, \mathbf{Z}_{n}$ is $2^{n}$, growing in the same way as for the Sierpinski iteration

$$
\left(\mathbf{Z}_{n}\right)=\left(\begin{array}{c}
\mathbf{1} \\
2 \cdot \mathbf{1}
\end{array}\right)=\left(\begin{array}{c}
\mathbf{Y}_{n-1} \\
\mathbf{Z}_{n-1}
\end{array}\right) \quad \text { with }\left(\begin{array}{l}
\mathbf{Y}_{0} \\
\mathbf{Z}_{0}
\end{array}\right)=\left(\begin{array}{l}
1 \\
2
\end{array}\right),
$$

as initial vector (Fig. 3), or the Thue-Morse iteration

$$
\left(\mathbf{Z}_{n}\right)=\left(\begin{array}{c}
\mathbf{1} \\
(-1) \cdot \mathbf{1}
\end{array}\right)\left(\begin{array}{c}
\mathbf{Y}_{n-1} \\
\mathbf{Z}_{n-1}
\end{array}\right) \text { with }\left(\begin{array}{c}
\mathbf{Y}_{0} \\
\mathbf{Z}_{0}
\end{array}\right)=\left(\begin{array}{c}
1 \\
-1
\end{array}\right)
$$

as initial vector [both use Eq. (8)].

To get acquainted with the formalism, it is illustrative to perform the first iterations explicitly. The first iteration, following Eqs. (8) and (9), reads

$$
\begin{gathered}
\mathbf{Y}_{1}=\left(\begin{array}{c}
\mathbf{Y}_{0} \\
\mathbf{Z}_{0}
\end{array}\right)=\left(\begin{array}{l}
1 \\
3
\end{array}\right), \\
\mathbf{Z}_{1}=\left(\begin{array}{c}
3 \mathbf{Y}_{0} \\
2 \mathbf{Y}_{0}+\mathbf{Z}_{0}
\end{array}\right)=\left(\begin{array}{l}
3 \\
5
\end{array}\right),
\end{gathered}
$$




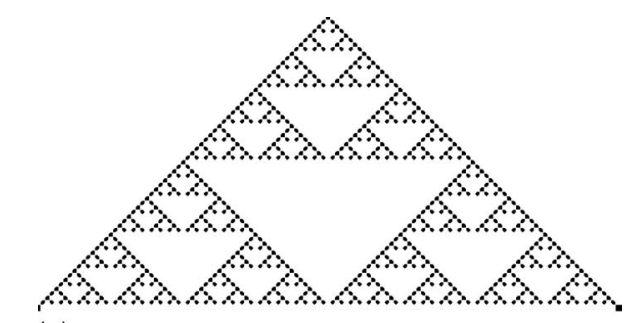

(a)

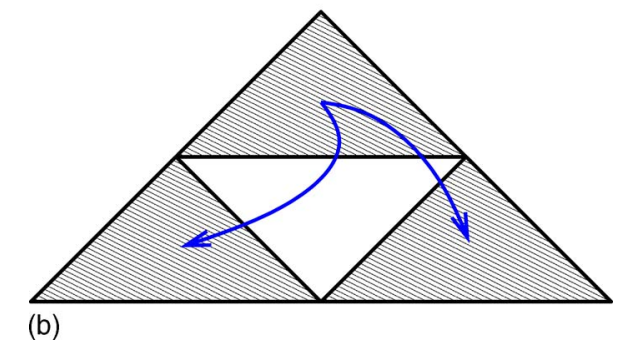

FIG. 3. (Color online) Illustration of the replication rule for rule 90 .

$$
\mathbf{Y}_{2}=\left(\begin{array}{l}
\mathbf{Y}_{1} \\
\mathbf{Z}_{1}
\end{array}\right)=\left(\begin{array}{l}
1 \\
3 \\
3 \\
5
\end{array}\right)
$$

Equivalently, Eq. (10) may be used, and the first two iterations read

$$
\left(\begin{array}{ll}
1 & 0 \\
0 & 1 \\
3 & 0 \\
2 & 1
\end{array}\right)\left(\begin{array}{l}
1 \\
3
\end{array}\right)=\left(\begin{array}{l}
1 \\
3 \\
3 \\
5
\end{array}\right)
$$

and

$$
\left(\begin{array}{llll}
1 & 0 & 0 & 0 \\
0 & 1 & 0 & 0 \\
0 & 0 & 1 & 0 \\
0 & 0 & 0 & 1 \\
3 & 0 & 0 & 0 \\
0 & 3 & 0 & 0 \\
2 & 0 & 1 & 0 \\
0 & 2 & 0 & 1
\end{array}\right)\left(\begin{array}{l}
1 \\
3 \\
3 \\
5
\end{array}\right)=\left(\begin{array}{c}
1 \\
3 \\
3 \\
5 \\
3 \\
9 \\
5 \\
11
\end{array}\right) .
$$

These iterations look formally similar to the Fibonacci or Lucas iteration

$$
\left(\begin{array}{c}
F_{n} \\
F_{n-1}
\end{array}\right)=\left(\begin{array}{ll}
1 & 1 \\
1 & 0
\end{array}\right)\left(\begin{array}{c}
F_{n-1} \\
F_{n-2}
\end{array}\right)
$$

Here $\left(F_{1}, F_{0}\right)^{T}=(1,0)^{T}$ defines the Fibonacci series and $\left(F_{1}, F_{0}\right)^{T}=(1,2)^{T}$ is the initial condition of the Lucas series. For the latter two, the length of the iterates is not growing. 


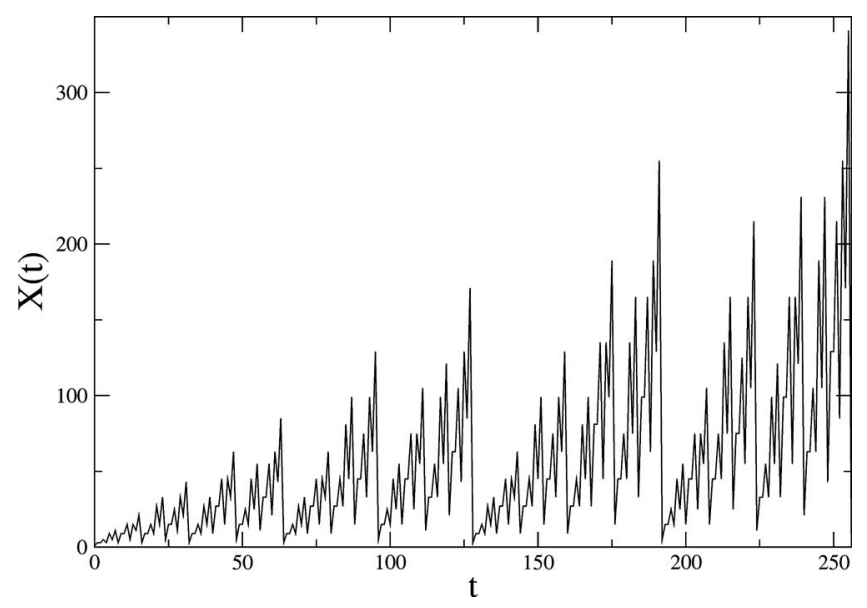

FIG. 4. Rule 150: Plot of $X(t)$ for the first 256 time steps.

Equation (6) or (10) equivalently, together with the initial condition $(1,3)^{T}$, generate $X(t)$ iteratively for all $t$. Formally this iteration is analogous to the Fibonacci iteration but acts on vectors of growing length within an infinite-dimensional vector space indexed by non-negative integer values.

This type of series should be distinguished from the $\left(r\right.$ th) hyper-Fibonacci series ${ }^{18}$ where $f_{l}$ $=2^{l-1}$ for $l=1, \ldots, r+1$ and $f_{l}=f_{l-1}+\cdots+f_{l-r+1}$ for $l>r+1$. On the other hand, the terminus generalized Fibonacci series is widely used for the ordinary Fibonacci or Lucas iteration with two arbitrary start values $f_{0}$ and $f_{1}$, where $f_{0}=0, f_{1}=1$ defines the Fibonacci series and $f_{0}=2, f_{1}=1$ defines the Lucas series; in fact, both can be used as (nonorthogonal) basis vectors of the linear space of generalized Fibonacci series. The $r=1$ hyper-Fibonacci series corresponds to a generalized Fibonacci series with $f_{0}=1, f_{1}=2$. Consequently, an iteration of the algebraic structure of Eq. (10) could be denoted as a generalized hyper-Fibonacci series.

Another observation is the partial self-similarity relation

$$
X\left(\sigma_{n} \cdots \sigma_{3}, 0, \sigma_{1}, \sigma_{0}\right)=X\left(\sigma_{1}, \sigma_{0}\right) \cdot X\left(\sigma_{n} \cdots \sigma_{3}\right)
$$

(leading zeros omitted in notation), i.e., the sequence generated by every second block of length four $\left(\sigma_{2}=0\right)$ factorizes into the first block $(1,3,3,5)$ and the whole sequence itself. A closed expression for $X\left(\sigma_{n} \cdots \sigma_{3}, 1, \sigma_{1}, \sigma_{0}\right)$ is, however, not known yet.

The first values of $X(t)=X\left(t_{1}+t_{2}\right)$ are listed in Table I (see Fig. 4).

\section{ITERATIVE SOLUTION VIA A FORMAL LANGUAGE REPLICATION RULE}

One can proceed similar to the Sierpinski (rule 90) case, where $X(t)$ can be generated from an initiator $a=(1)$ by the iterative replication $(a) \rightarrow(a, 2 \cdot a)$. For the Thue-Morse sequence, the iteration reads $(a) \rightarrow(a,(-1) \cdot a)$, see Refs. 19-21. However, for rule 150 again the Fibonacci-like structure comes into play, i.e., each iterate depends on two preceders for which one has to require $\operatorname{len}(a)=\operatorname{len}(b)$. Then the iteration is defined by

$$
(a, b) \rightarrow(a, b, 3 a, 2 a+b) .
$$

This is equivalent to Eq. (10).

\section{BLOCK-SUMS AND THE FIBONACCI SERIES}

Following the same geometrical argument as for the row sums, the sum $S_{n}=\sum_{i=0}^{2^{n}-1} X(i)$ is given by the iteration $S_{n}-S_{n-1}=S_{n-1}+4 S_{n-2}$ or 
TABLE II. The block sums $S_{n}$ for the first 18 time steps.

\begin{tabular}{lrrrrr}
\hline \hline$n$ & $S_{n}$ & $n$ & $S_{n}$ & $n$ & \multicolumn{1}{c}{$S_{n}$} \\
\hline 0 & 1 & 6 & 1344 & 12 & 1544192 \\
1 & 4 & 7 & 4352 & 13 & 4997120 \\
2 & 12 & 8 & 14080 & 14 & 16171008 \\
3 & 40 & 9 & 45568 & 15 & 52330496 \\
4 & 128 & 10 & 147456 & 16 & 169345024 \\
5 & 416 & 11 & 477184 & 17 & 548012032 \\
\hline \hline
\end{tabular}

$$
S_{n}=2 S_{n-1}+4 S_{n-2},
$$

and the first elements of the series are listed in Table II.

The matrix of the iteration in time-delayed coordinates,

$$
\left(\begin{array}{c}
S_{n} \\
S_{n-1}
\end{array}\right)=\left(\begin{array}{ll}
2 & 4 \\
1 & 0
\end{array}\right)\left(\begin{array}{c}
S_{n-1} \\
S_{n-2}
\end{array}\right),
$$

has the eigenvalues $\alpha_{1,2}=1 \pm \sqrt{5}$, indicating that it differs from the Fibonacci iteration matrix by an additional expansion factor of 2 , i.e., by a suitable transformation

$$
\left(\begin{array}{c}
S_{n} \\
2 S_{n-1}
\end{array}\right)=\left(\begin{array}{ll}
1 & 1 \\
1 & 0
\end{array}\right)\left(\begin{array}{c}
2 S_{n-1} \\
2^{2} S_{n-2}
\end{array}\right),
$$

it relates to the Fibonacci numbers in usual convention,

$$
\begin{aligned}
S_{n} & =F_{n+2} \cdot 2^{n} \\
& =\frac{1}{\sqrt{5}}\left[\left(\frac{1+\sqrt{5}}{2}\right)^{n}-\left(\frac{1-\sqrt{5}}{2}\right)^{n}\right],
\end{aligned}
$$

the latter following from Binet's formula. A "blockwise normalization" or detrended signal, as used in Ref. 8, can be achieved by subtracting

$$
N_{n}=\left(S_{n}-S_{n-1}\right) / 2^{n-1}=2 F_{n+2}-F_{n+1}=F_{n}+F_{n+2}
$$

from $X(t)$ for all $2^{n-1} \leqslant t \leqslant 2^{n}-1$. Then $X(t)-N_{\left\lfloor\log _{2} t\right]}$ defines a signal with a mean vanishing within each time interval $\left[0,2^{N}-1\right]$. The first values of this series (the Lucas numbers ${ }^{22}$ ) are $N_{0}, N_{1}, \ldots=1,3,4,7,11,18,29,47,76, \ldots$.

An immediate side result is that $S_{n}$ defines the total volume of sites in $(n, t)$ space having the value 1 , and this volume scales for $n \rightarrow \infty$ with the largest eigenvalue. Thus, if the time-doubling iteration is interpreted as a generation rule of the resulting self-similar fractal (rescaled to the unit interval), its Hausdorff-Besicovic dimension is given by $1+(\ln (1+\sqrt{5}) / 2) / \ln 2$.

\section{2D EXTENSION OF RULE 150}

The 2D extensions of rule 150 (and rule 90) as a mod 2 on the von Neumann neighborhood including (rule 150) or excluding (rule 90) the central node are shown in Fig. 32 in Ref. 3, and the time series from the simulated spatial dynamics is shown in Ref. 23. For rule 90 we have stated the solution in Ref. 9, i.e., the time series is just the square of the $1 \mathrm{D}$ case.

For the $2 \mathrm{D}$ rule 150 , 
(a)

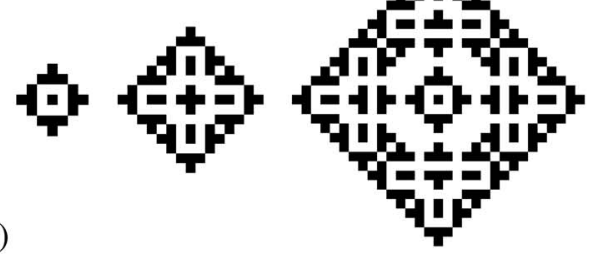

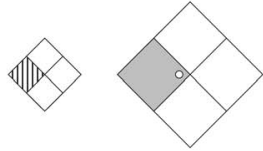

(b)

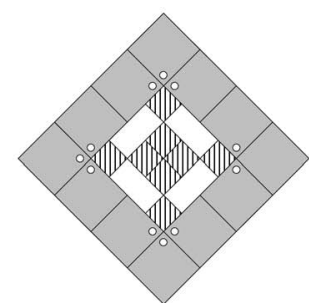

FIG. 5. Left: Time steps 3, 7, and 15 of the time evolution of the 2D extension of rule 150, started with a single seed. As these time steps are binary 11,111 , and 1111, the total numbers of active cells are just $\chi^{(2)}(2), \chi^{(2)}(3)$, and $\chi^{(2)}(4)$, respectively. Right: Illustration of the two-step replication rule; one quarter of the previous pattern (middle) is replicated 12 times (orientation indicated by circle) are one quarter of the pattern (shaded) is replicated 8 times. This replication corresponds to the composition of the bottom line in Fig. 1; the preceding time steps are obtained by stacking the respective (quarter) pyramids upon.

$$
x_{n, m}^{t+1}=\left[x_{n-1, m}^{t}+x_{n, m-1}^{t}+x_{n, m}^{t}+x_{n+1, m}^{t}+x_{n, m+1}^{t}\right] \bmod 2,
$$

the situation again is more complicated, but one can apply a similar procedure as above.

It is straightforward to see that the two-step iteration construction of Fig. 1 can be generalized to two dimensions (see Fig. 5), resulting in the iteration $(a, b) \rightarrow(a, b, 5 a, 2 a+3 b)$ or in the matrix notation

$$
\left(\begin{array}{c}
\mathbf{Y}_{n} \\
\mathbf{Z}_{n}
\end{array}\right)=\left(\begin{array}{cc}
\mathbf{1} & \mathbf{0} \\
\mathbf{0} & \mathbf{1} \\
5 \cdot \mathbf{1} & \mathbf{0} \\
2 \cdot \mathbf{1} & 3 \cdot \mathbf{1}
\end{array}\right)\left(\begin{array}{l}
\mathbf{Y}_{n-1} \\
\mathbf{Z}_{n-1}
\end{array}\right)
$$

The blockwise sums $\widetilde{S}_{0}, \widetilde{S}_{1}, \ldots=1,6,28,136,656, \ldots$ follow the iteration

$$
\left(\begin{array}{c}
\tilde{S}_{n} \\
\tilde{S}_{n-1}
\end{array}\right)=\left(\begin{array}{ll}
4 & 4 \\
1 & 0
\end{array}\right)\left(\begin{array}{l}
\tilde{S}_{n-1} \\
\tilde{S}_{n-2}
\end{array}\right)
$$

(eigenvalues $2 \pm \sqrt{8}$ ), which again is of the type of a generalized Fibonacci series. Again, the two-step iteration principle applies and the time series is obtained by replacing $\chi(n)$ in $(5)$ by $\chi^{(2)}(n)=1,5,17,61,217, \ldots{ }^{14,24,35,36}$ From the geometrical construction, one immediately has the recursion $\chi^{(2)}(n)=3 \chi^{(2)}(n-1)+2 \chi^{(2)}(n-2)$ with $\chi^{(2)}(0)=1$ and $\chi^{(2)}(1)=5$. This provides an analytical solution also for the $2 \mathrm{D}$ case.

\section{TOWARD CAs ON NETWORKS: RULE 150 ON BETHE LATTICES}

For many systems, especially in biology and social systems, the low-dimensional lattice topology does not provide a realistic description. For the general case of graphs including loops, degree correlations, and varying node degrees, it seems to be prohibitive to solve analytically the exact dynamics of rule 150 .

Restricting to degree-regular graphs without loops, however, allows us to proceed some steps and get an intuition about the possible total activity on a graph. For this, we consider a Bethe lattice where every node has $k$ nearest neighbors and again initialize the system with a single 1 on an arbitrary node. Considering the Bethe lattice as infinite, it does not matter at which node to start; on a real (and thus finite) substrate the consideration is valid only up to the time step when the evolving pattern reaches the surface nodes; thus in a simulation, one would start at the central node, which we denote as focal node $x_{0}$ here. 


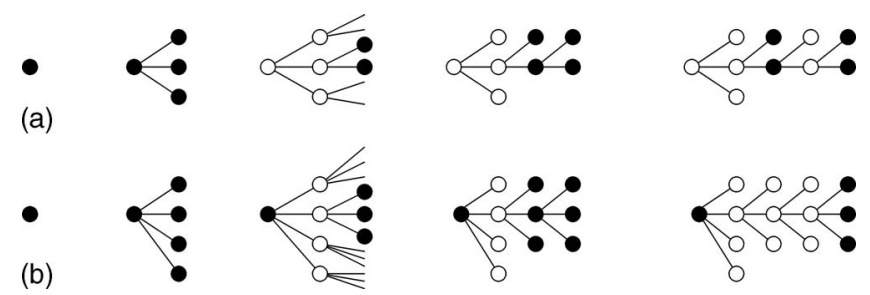

FIG. 6. Rule 150 on Bethe lattices. Only the active part of the lattice is shown, and only a part of the nodes in each generation is drawn. Shown are time steps $t=0,1,2,3,4$ with the focal node where the seed is placed on the left. The focal node has $k$ neighbors, whereas all nodes reached in subsequent states have one neighbor in the previous generation (left) and $k-1$ neighbors in the following generation. (a) Bethe lattice with an odd number of neighbors, $k=3$. After two steps, the inner nodes $\left(x_{0}, x_{1}\right)$ stay frozen in state 0 and the $k$ leaves perform a Sierpinski (rule 90) pattern ranging from $x_{2}$ to $x_{t+1}$. The shorthand notation of these states is $(1),(1,1),(0,0,1),(0,0,1,1),(0,0,1,0,1)$. (b) Bethe lattice with an even number of neighbors, $k=4$. Here, the focal node stays frozen in state 1 and all branches follow the right halfplane pattern of rule 150 (Fig. 1(a)). The shorthand notation of these states is $(1),(1,1),(1,0,1),(1,0,1,1),(1,0,0,0,1)$. Note that the 1D pattern in Fig. 1 is equivalent to a $k=2$ Bethe lattice, whereas an orthogonal 2D lattice cannot be represented as a Bethe lattice.

The dynamics then is remarkably simple, as the following inspection shows (Fig. 6). Due to the symmetry, all nodes $x_{n}$ that are $n$ steps away from $x_{0}$ behave identically; thus until time step $n$ we have only $x_{n}$ independent variables that can be nonzero and collect them into a (possibly infinitely long) vector $\left(x_{0}, x_{1}, x_{2}, \ldots\right)$. Two cases can be distinguished.

If $k$ is odd, the central node is connected to an odd number of nodes, and it follows the dynamics

$$
\begin{aligned}
& (0,0, \ldots) \rightarrow(0, \ldots), \\
& (0,1, \ldots) \rightarrow(1, \ldots), \\
& (1,0, \ldots) \rightarrow(1, \ldots), \\
& (1,1, \ldots) \rightarrow(0, \ldots)
\end{aligned}
$$

(which is equivalent to rule 102 or to rule 150 with the boundary condition of a fixed $x_{-1}=0$ ). All following $(n>0)$ nodes $x_{n}$ are connected to 1 node of type $x_{n-1}$ and to an even number of ( $k$ -1 ) nodes of type $x_{n+1}$, that is, the contributions of the outer nodes cancel out and $x_{n}$ does not depend on $x_{n+1}$. Here we have the rule table

$$
\begin{aligned}
& (\ldots, 0,0,0, \ldots) \rightarrow(\ldots, 0, \ldots), \\
& (\ldots, 0,0,1, \ldots) \rightarrow(\ldots, 0, \ldots), \\
& (\ldots, 0,1,0, \ldots) \rightarrow(\ldots, 1, \ldots), \\
& (\ldots, 0,1,1, \ldots) \rightarrow(\ldots, 1, \ldots), \\
& (\ldots, 1,0,0, \ldots) \rightarrow(\ldots, 1, \ldots), \\
& (\ldots, 1,0,1, \ldots) \rightarrow(\ldots, 1, \ldots), \\
& (\ldots, 1,1,0, \ldots) \rightarrow(\ldots, 0, \ldots),
\end{aligned}
$$




$$
(\ldots, 1,1,1, \ldots) \rightarrow(\ldots, 0, \ldots),
$$

being equivalent to rule 60 . This, hovever, is equivalent to rule 90 on a half lattice spacing (removing the trivial zeros within the Sierpinski triangle). The left border here stays fixed at $x_{2}$ $=1$, and the inner $k$ nodes $\left(x_{0}\right.$ and $\left.x_{1}\right)$ are frozen to zero from the second time step onward.

If $k$ is even, the central node is connected to an even number of nodes $x_{1}$,

$$
\begin{aligned}
& (0,0, \ldots) \rightarrow(0, \ldots), \\
& (0,1, \ldots) \rightarrow(0, \ldots), \\
& (1,0, \ldots) \rightarrow(1, \ldots), \\
& (1,1, \ldots) \rightarrow(1, \ldots),
\end{aligned}
$$

which is the rule table of rule 204 when we formally add the noncontributing $x_{-1}$. Hence the focus node stays frozen, i.e., $\forall_{t} x_{0}(t)=1$, same as in the $1 \mathrm{D}$ lattice version of rule 150 . All following nodes of type $x_{n}$ are connected to one node of type $x_{n-1}$ and to an odd number of $(k-1)$ nodes of type $x_{n+1}$, which in the modulo is equivalent to having one node on the right. Thus we have the rule table

$$
\begin{aligned}
& (\ldots, 0,0,0, \ldots) \rightarrow(\ldots, 0, \ldots), \\
& (\ldots, 0,0,1, \ldots) \rightarrow(\ldots, 1, \ldots), \\
& (\ldots, 0,1,0, \ldots) \rightarrow(\ldots, 1, \ldots), \\
& (\ldots, 0,1,1, \ldots) \rightarrow(\ldots, 0, \ldots), \\
& (\ldots, 1,0,0, \ldots) \rightarrow(\ldots, 1, \ldots), \\
& (\ldots, 1,0,1, \ldots) \rightarrow(\ldots, 0, \ldots), \\
& (\ldots, 1,1,0, \ldots) \rightarrow(\ldots, 0, \ldots), \\
& (\ldots, 1,1,1, \ldots) \rightarrow(\ldots, 1, \ldots) .
\end{aligned}
$$

This is precisely rule 150 on a 1D lattice (which is a Bethe lattice with $k=2$ ).

In both cases, the total activity can be written as

$$
X(t)=x_{0}+k\left[x_{1}+\sum_{i=2}^{t}(k-1)^{i-1} x_{i}\right] .
$$

While the dynamics of the degrees of freedom are identical for all even and for all odd Bethe lattices, respectively, the total number of active cells of course increases with $k$. For $k=2,3,4$ we have $\mathrm{e}^{25,26}$

$$
\begin{gathered}
X_{2}^{B}=1,3,3,5,3,9, \ldots, \\
X_{3}^{B}=1,4,6,18,30,90,102,306,510,1530,1542, \ldots,
\end{gathered}
$$




$$
X_{4}^{B}=1,5,13,49,109,473,1081,4037,8749, \ldots .
$$

Due to the high symmetry and the absence of loops, still a regular pattern is obtained. The growth of $X(t)$, which is dominated by the nodes at the surface of the pattern (due to their multiplicity), gives an estimate of the scaling of increase which could occur on more general graphs; here we especially observe the distinct nature of nodes of odd and even degree. For special time steps, only two types of nodes are nonzero, and explicit expressions can be given. If $k$ is odd, we have ( $N$ $\geqslant 1)$

$$
X_{k}^{B}\left(t=2+2^{N}\right)=k+\frac{k-1}{k}(k-1)^{t} .
$$

For even $k, N \geqslant 1$, the corresponding expression reads

$$
X_{k}^{B}\left(t=2^{N}\right)=1+\frac{k}{k-1}(k-1)^{t} .
$$

An application of the dynamics as depicted here could be a consistency or checksum version of gossip or opinion spread, on a topology generated by a process where each agent can send a chain letter to a fixed number of other people, and continues thereafter to communicate on the established links.

\section{CONCLUSIONS AND OUTLOOK}

The self-similarity structure of the rule 150 ECA generated space-time fractal is qualitatively different from the Sierpinski triangle generated by rule 90 . While the iteration itself generalizes the concept of a Fibonacci iteration to vectors of growing dimension, the blockwise sum exactly is given by the Fibonacci series multiplied by a scaling factor $2^{n}$. In this paper, the generating iterations for the 1D and 2D lattice were given, as well as for Bethe lattices of arbitrary coordination number. Besides the beauty of the symmetries of the dynamics, the results have the practical advantage of reducing the computational complexity of the considered time series: the iteration rule for the total activity derived here allows us to compute the total activity without simulating the spatial dynamics and thus considerably eases the numerical computation.

Previously, CAs have mainly raised interest as models for universal computation, for biological and chemical pattern formation, and as paradigmatic models for complex multiscale phenomena, which did and still do complement numerical progress. Recent attention to CAs stems from different directions, as from systematically relating CA to other methods, e.g., by coarse graining, ${ }^{27}$ considering criticality and universality classes, ${ }^{9,28-30}$ as models for $1 / f^{\alpha}$ fluctuations, ${ }^{8}$ also in astrophysical accretion phenomena, ${ }^{31}$ in urban growth, ${ }^{32}$ in technical applications as efficient VLSI device test, ${ }^{33}$ or from relations between integer sequences and graphs. ${ }^{34-36}$ Currently many models of spatial evolutionary dynamics, ${ }^{37-41}$ which in a zero-temperature limit and with parallel update dynamics resemble CAs on a lattice, are transferred onto graphs, ${ }^{42}$ which often more realistically reflect the interaction topology, especially in social dynamics. However, also in biological systems CA models re-emerge. The dynamics of CAs on graphs is of current interest especially regarding metabolic, regulatory, and boolean networks. ${ }^{43-46}$ As simplified models are a necessary means to detect universal features in large networks, ${ }^{47} \mathrm{CA}$ dynamics can be a valuable null model to complement other levels of description.

\footnotetext{
${ }^{1}$ P. Bak, C. Tang, and K. Wiesenfeld, Phys. Rev. Lett. 59, 381 (1987), Phys. Rev. A 38, 364 (1988).

${ }^{2}$ H. J. Jensen, Self-organized Criticality (Cambridge University Press, Cambridge, 1998).

${ }^{3}$ S. Wolfram, Physica D 10, 1 (1984); Nature (London) 311, 419 (1984).

${ }^{4}$ S. Wolfram, Rev. Mod. Phys. 55, 601 (1983).

${ }^{5}$ J. Xu and A. Hübler, Phys. Rev. B 67, 184202 (2003).

${ }^{6} \mathrm{R}$. Mosseri, e-print arXiv:0801.2860.

${ }^{7}$ M. Gardner, Sci. Am. 223(10), 120 (1970).

${ }^{8}$ J. C. Claussen, J. Nagler, and H. G. Schuster, Phys. Rev. E 70, 032101 (2004).

${ }^{9}$ J. Nagler and J. C. Claussen, Phys. Rev. E 71, 067103 (2005).
} 
${ }^{10}$ C. Tang, K. Wiesenfeld, P. Bak, S. Coppersmith, and P. Littlewood, Phys. Rev. Lett. 58, 1161 (1987).

${ }^{11}$ Y. Oono and M. Kohmoto, Phys. Rev. Lett. 55, 2927 (1985).

${ }^{12}$ Closed solution from generating functions: A straightforward expression for the time evolution of $x_{n}^{t}$ by rule 150 for the initial condition of a single 1 at $n=0$ and $t=0$ is defined by the coefficients of $P_{t}(x):=\left(1+x+x^{2}\right)^{t}$ (Ref. 4), or Gegenbauer functions, and performs the mod 2 operation afterward, i.e., $P_{t}(x)=\sum_{n=0}^{2 t} p_{n}^{(t)} x^{n}$ and $x_{n}^{t}=p_{n+t}^{(t)} \bmod 2$. From $P_{t}(x)$, the coefficients can be extracted via the method of generating function, giving

$$
x_{n}^{t}=\left[\left.\frac{\partial^{n+t}}{\partial x^{n+t}} P_{t}(x)\right|_{x=0}\right] \bmod 2 .
$$

Hovever contrary to the case of rule 90, generating the Sierpinski gasket, and where the cofficients of $(1+x)^{t}$ simply are the binomial coefficients, this does not lead to a closed expression for $X(t)$ rule 150 .

${ }^{13}$ The iteration for $\chi(n)$ on p. 614 in Ref. 4 seems to be a typograpical error.

${ }^{14}$ N. J. A. Sloane, Not. Am. Math. Soc. 50, 912 (2003).

${ }^{15}$ N. J. A. Sloane, OEIS Sequence A001045 (http://www.research.att.com/ njas/sequences/A001045).

${ }^{16}$ Of order $O\left(t(\log t)^{3}\right)$, optimized $O(t \log t)$; the spatial simulation is $O\left(t^{2}\right)$; iteration (10) is $O(t)$ as the matrices are diagonal.

${ }^{17}$ G. W. Grossmann, Fibonacci Quarterly 35.1, 206 (1997).

${ }^{18}$ J. C. Alexander, The entropy of the Fibonacci code (1989) (available at http://www.cwru.edu/artsci/math/alexander/ abstracts/fibabs.html).

${ }^{19}$ A. S. Pikovsky, M. A. Zaks, U. Feudel, and J. Kurths, Phys. Rev. E 52, 285 (1995).

${ }^{20}$ M. A. Zaks, A. S. Pikovsky, and J. Kurths, Phys. Rev. Lett. 77, 4338 (1996).

${ }^{21}$ M. A. Zaks and A. V. Straube, Phys. Rev. Lett. 89, 244101 (2002).

${ }^{22}$ N. J. A. Sloane, OEIS Sequence A000204 (http://www.research.att.com/ njas/sequences/A000204).

${ }^{23}$ N. H. Packard and S. Wolfram, J. Stat. Phys. 38, 901 (1985).

${ }^{24}$ N. J. A. Sloane, OEIS Sequence A007483 (http://www.research.att.com/ njas/sequences/A007483).

${ }^{25}$ J. C. Claussen, OEIS Sequence A138276 (http://www.research.att.com/ njas/sequences/A138276).

${ }^{26}$ J. C. Claussen, OEIS Sequence A138277 (http://www.research.att.com/ njas/sequences/A138277).

${ }^{27}$ N. Israeli and N. Goldenfeld, Phys. Rev. Lett. 92, 074105 (2004).

${ }^{28}$ V. C. Barbosa, F. M. N. Miranda, and M. C. M. Agostini, Bull. Am. Astron. Soc. 32, 44 (2006).

${ }^{29}$ M. T. Matache and J. Heidel, Phys. Rev. E 69, 056214 (2004).

${ }^{30}$ M. T. Matache and J. Heidel, Phys. Rev. E 71, 026232 (2005).

${ }^{31}$ T. Kawaguchi, S. Mineshige, M. Machida, and R. Matsumoto, Publ. Astron. Soc. Jpn. 52, 98 (2000).

${ }^{32}$ D. P. Ward, A. T. Murray, and S. R. Phinn, Comput. Environ. Urban Syst. 24, 539 (2000).

${ }^{33}$ S. H. U. Tezuka and M. Fushimi, Contemp. Math. 168, 363 (1994).

${ }^{34}$ P. Barry, J. Integer Sequences 10, 07.4 .8 (2007).

${ }^{35}$ A. Burstein, S. Kitaev, and T. Mansour, arXiv:math/0310379.

${ }^{36}$ R. K. Guy and W. O. J. Moser, Fibonacci Quarterly 34, 152 (1996).

${ }^{37}$ M. A. Nowak and R. M. May, Nature (London) 359, 826 (1992).

${ }^{38}$ K. Lindgren and M. Nordahl, Physica D 75, 292 (1994).

${ }^{39}$ A. V. M. Herz, J. Theor. Biol. 169, 65 (1994).

${ }^{40}$ A. Traulsen and J. C. Claussen, Phys. Rev. E 70, 046128 (2004).

${ }^{41}$ G. Szabó, A. Szolnoki, and G. A. Sznaider, Phys. Rev. E 76, 051921 (2007).

${ }^{42}$ G. Szabo and G. Fath, Phys. Rep. 446, 97 (2007).

${ }^{43}$ B. Drossel, arXiv:0706.3351.

${ }^{44}$ A. Szejka and B. Drossel, Eur. Phys. J. B 56, 373 (2007).

${ }^{45}$ C. Marr and M. T. Hütt, Physica A 354, 641 (2005).

${ }^{46}$ K. Klemm and S. Bornholdt, Proc. Natl. Acad. Sci. U.S.A. 102, 18414 (2005).

${ }^{47}$ S. Bornholdt, Science 310, 449 (2005). 